\title{
Immunological biomarkers in childhood and adolescence obesity
}

\author{
A. Hernández ${ }^{1}$, T. Pozo-Rubio ${ }^{1}$, A. Gheorghe ${ }^{1}$, L. E. Díaz ${ }^{1}$, F. Pérez de Heredia ${ }^{1}$, P. De Miguel ${ }^{2,3,4}$, \\ E. Gonzalez ${ }^{2,3}$, C. M. Aguilera ${ }^{5}$ O. D. Rangel ${ }^{5}$, M. Gil-Campos ${ }^{6}$, K. Flores ${ }^{6}$, J. Maldonado ${ }^{7}$, \\ R. Hoyos ${ }^{7}$, A. Gil ${ }^{5}$, L. Moreno ${ }^{2}$ and A. Marcos ${ }^{1}$ \\ ${ }^{1}$ Immunonutrition Research Group, ICTAN, CSIC, Madrid, Spain, ${ }^{2}$ GENUD "Growth, Exercise, Nutrition and \\ Development" Research Group, University of Zaragoza, Spain, ${ }^{3}$ Dept. Physiotherapy and Nursing, Faculty of Health \\ Sciences, University of Zaragoza, Spain, ${ }^{4}$ Dept. Paediatrics, Radiology and Physical Medicine, Faculty of Medicine, \\ University of Zaragoza, Spain, ${ }^{5}$ Dept. Biochemistry and Molecular Biology, Institute of Nutrition and Food Technology, \\ University of Granada, Spain, ${ }^{6}$ Paediatric Service, University Hospital Reina Sofía, Córdoba, Spain and ${ }^{7}$ Paediatric \\ Service, University Hospital Virgen de las Nieves, Granada, Spain
}

\begin{abstract}
Obesity and metabolic syndrome are shown to be related to changes in the immune system, both at the cellular and humoral levels. Indeed, higher T lymphocyte counts have been associated with greater body mass index (BMI) ${ }^{(1)}$, and total leukocyte count is positively correlated with markers of metabolic syndrome ${ }^{(2)}$. The aim of this study was to detect possible interactions between risk of metabolic syndrome and immunological biomarkers. To this end, relationships between immunological markers and BMI, total cholesterol, HDL-c and LDL-c, triglycerides (TGs) and C-reactive protein (CRP), were evaluated in a sample of obese children (7-10.9 y, $n=58)$ and adolescents (11-16y, $n=132$ ) from Granada, Córdoba and Zaragoza (the PRONAOS study). Among the immunological markers, CD19 (B cells), CD3 (T cells), CD4 (helper T cells), CD8 (cytotoxic T cells), CD45RA (naïve cells) and CD45RO (memory cells) were analyzed by flow cytometry and serum immunoglobulin levels (IgA, IgG2, IgG3, IgG4, total IgG, IgE and IgM) by Cytometric Bead Array (Becton Dickinson) and C-reactive protein (CRP) by nephelometry. Spearman correlation test was used to analyze associations between these variables. BMI showed a positive correlation with CRP, in both children and adolescents. Only in children, BMI was

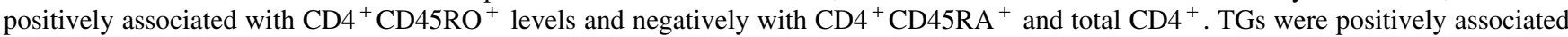
with absolute counts and percentages of $\mathrm{B}$ cells $\left(\mathrm{CD} 19^{+}\right)$in the whole population, and with $\mathrm{CD} 3{ }^{+} \mathrm{CD} 45 \mathrm{RA}{ }^{+}$in adolescents. In addition, TGs showed a significant correlation with IgE, which was positive in children and negative in adolescents. Total cholesterol showed a positive association with $\mathrm{CD}^{+} \mathrm{CD}^{+}$and negative with total $\mathrm{CD} 3^{+}$in children, while LDL-c was positively correlated with $\mathrm{CD} 4{ }^{+} \mathrm{CD} 45 \mathrm{RO}^{+}$. In conclusion, our results show that the immune system might suffer from an age-related adaptation with regard to the metabolic syndrome risk observed in these obese children and adolescents. Therefore, these immunological markers could be used as potential biomarkers for obesity and related metabolic alterations in children and adolescents in further studies.
\end{abstract}

This work was supported by PROYECTO CENIT-PRONAOS (PRONAOS Study, CDTI 20081114 and Biosearch.

1. Zaldivar F, McMurray RG, Nemet $\mathrm{D}$ et al. (2006) Int J Obes 30, 906-911.

2. Wu CZ, Hsiao FC, Lin JD et al. (2010) Acta Diabetologica 47, 65-71. 